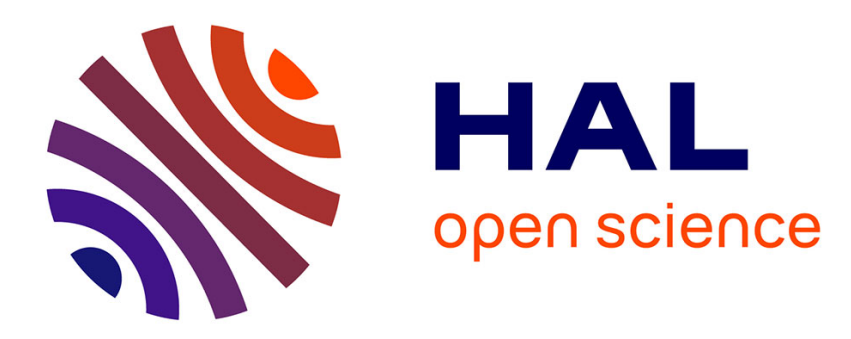

\title{
Maçons turcs, un exemple de stratification ethnique dans l'économie bretonne
}

\author{
Anne Yvonne Guillou, Martine Wadbled
}

\section{To cite this version:}

Anne Yvonne Guillou, Martine Wadbled. Maçons turcs, un exemple de stratification ethnique dans

l'économie bretonne. Hommes \& migrations, 2006, mars-avril 2006 (1260), pp.81-95. halshs-00322650

\section{HAL Id: halshs-00322650 \\ https://shs.hal.science/halshs-00322650}

Submitted on 18 Sep 2008

HAL is a multi-disciplinary open access archive for the deposit and dissemination of scientific research documents, whether they are published or not. The documents may come from teaching and research institutions in France or abroad, or from public or private research centers.
L'archive ouverte pluridisciplinaire HAL, est destinée au dépôt et à la diffusion de documents scientifiques de niveau recherche, publiés ou non, émanant des établissements d'enseignement et de recherche français ou étrangers, des laboratoires publics ou privés. 


\title{
Maçons turcs, un exemple de stratification ethnique dans l'économie bretonne
}

\author{
Originaires des régions pauvres de Turquie, les premiers immigrés installés en Bretagne \\ se sont taillés une solide réputation de maçons courageux et travailleurs. Le regroupement familial, \\ les crises successives du bâtiment les ont poussés à devenir artisans indépendants et donc en \\ concurrence alors qu'entre eux la solidarité est grande. La fragilité de leur entreprise, confrontée \\ à des problèmes de gestion, pose la question de l'intégration socio-économique de cette "enclave \\ ethnique" dans l'économie bretonne.
}

La figure archétypale du Gastarbeiter ("travailleur invité") en Allemagne ou de l'ouvrier immigré en France a fait long feu. Cet ouvrier venu de sa lointaine campagne d'Afrique du Nord ou de Turquie, sans sa famille, après la Seconde Guerre mondiale, jouissant de droits limités dans son pays d'accueil, peu ou pas intégré sur le plan politique et social et, surtout, censé repartir à la fin de son contrat de travail, n'existe plus. Le regroupement familial l'a durablement fixé sur le sol des pays d'émigration, européens principalement, et les formes d'intégration socio-économiques se sont diversifiées, se sont complexifiées. Dans un contexte de diminution du nombre d'emplois industriels sur le marché secondaire, incapables d'atteindre le marché primaire de l'emploi - celui des emplois stables, les mieux rémunérés et les plus valorisés -, les migrants ont peu à peu créé des activités de commerçants ou d'entrepreneurs, forgeant ainsi une économie ethnique (ethnic business) s'appuyant, à divers degrés, sur leurs groupes ethniques dont les membres sont employés et clients. Aux États-Unis par exemple, où le phénomène est mieux étudié par les sociologues, les "ethniques"(1) occupant des emplois indépendants sont proportionnellement plus nombreux que les majoritaires depuis les années quatre-vingt. Ces domaines d'activités, que les sociologues appellent des "niches ethniques", constituent une réponse largement utilisée à l'exclusion du marché général du travail, les immigrés et leurs descendants occupant des mini-secteurs économiques laissés vacants par les membres des groupes majoritaires. Ils s'inscrivent en général dans des créneaux où il existe une demande spécifique de produits de la part de consommateurs "ethniques", comme les épiceries. Ces petits entrepreneurs peuvent aussi être pourvoyeurs de main-d'œuvre et de logements pour par Anne Guillou, sociologue et anthropologue, chargée d'études à l'Addras, membre associé de l'équipe d'accueil "Laboratoire anthropologie et sociologie" de Rennes 2, chargée d'enseignement à l'université de Haute-Bretagne-Rennes 2

et Martine Wadbled,

sociologue, chargée d'études à l'Addras, membre de

I'Urmis (unité de recherche

migrations et société), membre associé de l'unité mixte de recherche Reso (université Haute-Bretagne-CNRS), chargée d'enseignement à l'université de

Haute-Bretagne-Rennes 2.
1)- Le terme "ethnique", dans la perspective de la sociologie des relations inter-ethniques, s'oppose à ceux de "majoritaire", "global", "natif". Il entend rappeler que les identités et les statuts des immigrés et de leurs descendants (ou ceux qui sont perçus comme tels) sont construits dans un rapport social inégalitaire avec les "natifs" (ou perçus comme tels). 
leurs "co-ethniques" récemment arrivés, jouant ainsi un rôle d'intermédiaire avec la société globale ${ }^{(2)}$.

Les migrants turcs en Bretagne ${ }^{(3)}$ répondent quant à eux au schéma de l"'enclave ethnique", pour reprendre le terme d'Alejandro Portes et son équipe ${ }^{(4)}$. L'enclave ethnique, en tant que forme d'intégration à l'économie du pays d'accueil, suppose bien, tout comme la niche ethnique, la concentration spatiale d'un groupe de migrants et sa stratification interne. Mais, à la différence de l'ethnic business qui, lui, est essentiellement tourné vers les co-ethniques, l'enclave ethnique forme au contraire un sous-secteur de l'économie générale, c'est-à-dire qu'elle s'adresse à tous et pas seulement aux co-ethniques. Ainsi, les emplois masculins turcs en Bretagne ont deux caractéristiques majeures, d'une part une très forte intégration dans le secteur important du bâtiment dont ils sont devenus un rouage indispensable dans le domaine de la pose de parpaings - et à un moindre degré, du coffrage - et, d'autre part, la très forte organisation intra-ethnique de ce secteur.

\section{L'enclave ethnique du bâtiment}

Les Turcs de Bretagne sont ainsi essentiellement ouvriers et artisans : 286 artisans et 900 ouvriers de cette nationalité sont recensés en 1999. La majorité (65 \%) est employée dans le bâtiment (le gros œuvre), ce qui constitue une particularité bretonne car les Turcs ne sont que $27 \%$ dans ce secteur, au plan national. Le bâtiment est en effet un domaine clé de l'économie bretonne puisqu'il occupe 72400 actifs (dont 11000 artisans travaillant seuls et 7800 artisans employant des salariés ainsi que 1100 chefs d'entreprise de type PME). Le secteur représente $9 \%$ du total des emplois salariés bretons (public et privés confondus) $)^{(5)}$. Les Turcs sont donc bien ancrés dans cette activité, même s'ils

2)- La première à avoir décrit ce phénomène d'intermédiaire est E. Bonacich, "A Theory of Middlemen Minorities", American Sociological Review, 1973, 38, pp. 583-594.

3)- Lanalyse présentée dans cet article provient d'une étude sociologique plus générale des migrations turques en Bretagne. Elle se base sur une enquête de terrain de neuf mois (20022003), réalisée dans trois villes bretonnes à des fins comparatives, Rennes (Ille-et-Vilaine), Vannes (Morbihan) et Quimper (Finistère). Elle a consisté en entretiens auprès de 46 familles turques et de 16 personnes ressources turques (représentants d'associations, religieux, enseignants, interprètes), ainsi que d'une centaine d'acteurs de la société française (municipalité et région, services sociaux, enseignants, associations, voisins, policiers, prêtres, médecins, etc.). L'enquête qualitative a été complétée par des observations de manifestations publiques et privées liées à la vie des familles turques en Bretagne, ainsi que par le recueil de données statistiques de seconde main (Insee) ou de première main (dépouillement du fichier des résidents d'un foyer de travailleurs sur vingt ans, demandes de permis de construire, etc.). Le rapport complet est téléchargeable sur le site $h t t p: / / w w w . a d d r a s . c o m$. Cette recherche a été réalisée par l'Addras, grâce à un cofinancement du Fasild et d'autres institutions bretonnes (DDE Morbihan, Ville de Rennes, Ville de Vannes, Ville de Quimper, conseil général du Finistère, CAF du Finistère).

4)- Voir notamment A. Portes and L. Jensen, "What's an Ethnic Enclave ? The Case for Conceptual Clarify", American Sociological Review, 1987, 52, pp. 768-771.

5)- Cellule économique de Bretagne, Le bâtiment en Bretagne, janvier 2003. 


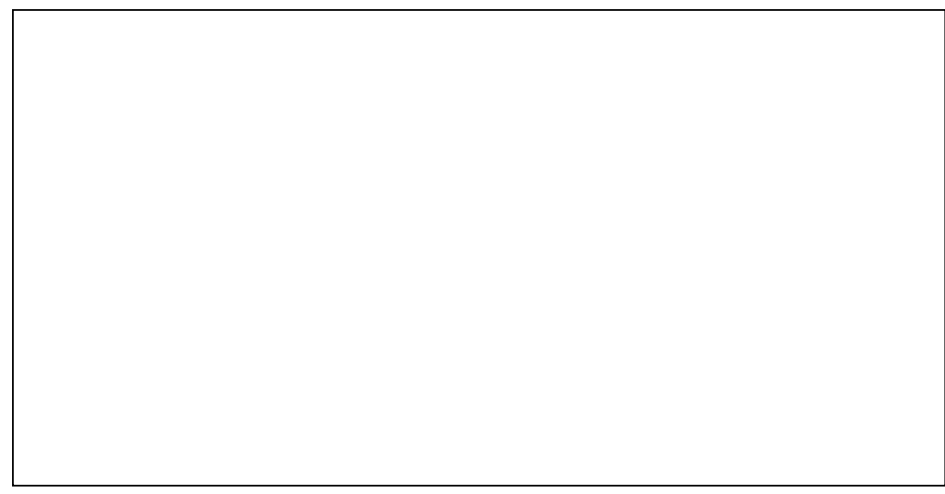

ne sont pas aussi nombreux que leur visibilité le fait généralement croire, à cause de leur concentration géographique ("Il n'y a plus que des Turcs dans ce secteur", a-t-on entendu souvent pendant l'enquête). Cette concentration s'observe par exemple au Blosne, quartier de la périphérie Sud de Rennes. Parmi les 381 entreprises actives dans tous les domaines, les Turcs sont présents exclusivement et sans exception dans le secteur de la construction. Parmi les 100 entreprises de construction recensées, 43 sont dirigées par des Turcs contre 7 par des Maghrébins et le reste par des Français ${ }^{(6)}$. Les statistiques donnent des proportions similaires à Quimper où 14 des 25 entreprises de maçonnerie créées entre janvier et décembre 2001 étaient dirigées par des Turcs ${ }^{(7)}$. Nous nous proposons donc d'étudier ici les mécanismes de cette enclave ethnique.

L'arrivée de "Turcs"(8) en Bretagne s'inscrit dans un contexte de migrations récentes, la région ayant été pendant longtemps davantage un pôle d'émigration que d'immigration. C'est à partir des années soixante-dix que les travailleurs turcs commencent à arriver ; mouvement s'accentuant dans les années quatre-vingt avec les regroupements familiaux. L'immigration étrangère, restreinte au regard de celle d'autres régions représente un peu plus de $1 \%$ de la population totale (5,7 \% au niveau national), avec des variations notables entre Rennes, où l'on observe la plus forte proportion d'étrangers de la région (4\% de la population) et d'autres villes comme Quimper ou Vannes, par exemple, où, toutes nationalités confondues, les étrangers comptent moins de $2 \%$ de l'ensemble de la population.

Les Turcs représentent un peu plus de $10 \%$ des étrangers de la région et $5,8 \%$ des immigrés ${ }^{(9)}$. Il est à noter que la présence des ressortissants turcs parmi les étrangers varie selon les villes : ils représentent, par exemple, près du tiers des étrangers à Quimper, la moitié à Vannes et 12,5 \% à Rennes. Dans les deux premières villes, ils occupent la première place au sein de la population étrangère, alors qu'à Rennes, ils sont en deuxième position derrière les Marocains. Globalement, la population issue de Turquie en Bretagne (définie ici
6)- Chambre des métiers et de l'artisanat d'Ille-et-Vilaine.

7)- Chambre des métiers et de l'artisanat du Finistère.

8)- Par le terme "turc", nous entendons l'ensemble aux contours flous -

des individus qui, d'une façon ou d'une autre, entretiennent un lien avec la Turquie,

se sentent, se disent ou sont définis comme "turcs" (migrants turcs et leurs enfants nés en France, minorités ethniques de Turquie, nationaux turcs comme naturalisés Français)

9)- Selon l'Insee : "Personnes nées étrangères dans un pays étranger. Ainsi une personne continue d'appartenir à la population immigrée même si elle devient française; on parlera alors d'immigrés, Français par acquisition". 
comme l'ensemble comprenant des nationaux turcs, et des personnes de nationalité française, par acquisition ou à la naissance) peut être estimée aujourd'hui en 2006 entre 4000 et 5000 personnes.

\section{Originaires des régions rurales pauvres}

Les caractéristiques socio-économiques des migrants varient selon les périodes d'arrivée : aux travailleurs des années soixante-dix, ruraux, peu scolarisés et sans qualification professionnelle succèdent, à partir des années quatre-vingt, des hommes et femmes d'origine plus urbaine et nantis d'un bagage scolaire plus élevé ou d'une formation professionnelle, venus le plus souvent par le mariage avec un(e) Turc(que) de France ou dans le cadre de l'asile politique. L'arrivée des premiers s'est effectuée dans le cadre d'une migration de travail masculine, de maind'œuvre non qualifiée dans les secteurs du bâtiment et de l'agroalimentaire. Les seconds, par commodité mais aussi du fait de la difficulté de trouver un emploi correspondant à leur formation, rejoignent leurs aînés dans ces secteurs. À côté de l'emploi masculin, un nombre croissant de femmes (jeunes) travaille dans l'agroalimentaire (Redon, Vannes, Briec, Quimper), activité économique importante dans la région. À une échelle beaucoup plus réduite - mais le phénomène est en augmentation -, la restauration rapide ("kebabs") constitue l'autre pôle des activités des Turcs en Bretagne.

Ces personnes sont originaires, principalement, des régions rurales et pauvres de l'est de la Turquie : une proportion non négligeable de personnes interviewées dans le cadre de l'enquête vient de la région de la mer Noire (Trabzon, Gumusane, Bayburt, Samsun ou surtout de villages aux environs de ces villes). Certaines familles, en plus petit nombre, sont issues de la zone plus continentale (Erzurum, Erzincan, Elazig), de villes et villages situés à l'est d'Ankara (Çorum et Yozgat), du Centre-Sud du pays (Konya). Les régions du Sud (Gaziantep et Hatay) et de l'Ouest sont très peu représentées, de même que les villes d'Istanbul et d'Izmir. Les

\begin{tabular}{|c|c|c|c|c|c|c|c|c|}
\hline & Bretagne & $\%$ & $\begin{array}{l}\text { Ille-et- } \\
\text { Vilaine } \\
\quad 35\end{array}$ & $\begin{array}{c}\text { Côtes } \\
\text { d'Armor } \\
22\end{array}$ & $\begin{array}{c}\text { Finistère } \\
29\end{array}$ & $\begin{array}{c}\text { Morbihan } \\
56\end{array}$ & France & $\%$ \\
\hline Agriculture & 30 & 2,67 & 12 & 06 & 08 & 04 & 2231 & 3,88 \\
\hline Industrie & 171 & 15,25 & 22 & 25 & 104 & 20 & 17915 & 31,17 \\
\hline Construction & 733 & 65,40 & 276 & 47 & 141 & 269 & 15613 & 27,18 \\
\hline Tertiaire & 147 & 13,11 & 42 & 12 & 44 & 49 & 14674 & 25,53 \\
\hline Commerce & 40 & 3,57 & 28 & 00 & 4 & 08 & 7036 & 12,24 \\
\hline total & 1121 & 100 & 380 & 90 & 301 & 350 & 57469 & 100 \\
\hline
\end{tabular}

Source : Insee, RP 99, Ex Compl. 
familles qui viennent des grandes villes (Istanbul, Izmir, Ankara, Konya) ont une origine géographique familiale généralement plus orientale et l'installation dans ces villes s'est effectuée lors d'une première migration vers l'Ouest, au cours des années soixante.

Le sex-ratio penche légèrement en faveur des hommes dans la tranche des 20-64 ans (56 \% des ressortissants turcs, recensement de 1999). C'est là l'écho du caractère masculin de la migration dans les années soixante-dix, et ce déséquilibre disparaît chez les plus jeunes, souvent nés en France. Les femmes sont cependant plus nombreuses chez les plus de 65 ans, à cause de la mortalité masculine précoce, due aux conditions de vie éprouvantes des premiers travailleurs migrants. La population issue de Turquie est généralement plus jeune que la population bretonne globale avec des écarts importants aux deux extrémités de l'échelle des âges (34\% de Turcs de moins de 20 ans contre $22 \%$ dans la population totale ; $1 \%$ de Turcs de plus de 65 ans contre $17 \%$ dans la population totale). La forte proportion d'enfants et d'adolescents révèle un taux de fécondité qui était, jusqu'à présent, plus élevé que dans la population globale mais qui évolue désormais dans le sens d'une limitation des naissances chez les couples plus jeunes, enfants des premiers migrants.

\section{Victimes de représentations négatives}

Dans la migration, on constate certains types de regroupements, en fonction de la région par exemple. Des familles élargies issues d'un même village, les hemseri peuvent constituer des noyaux de sociabilité privilégiés (plusieurs familles, à Rennes, par exemple, du village de Kelkit, les "Kelkitli" ; à Vannes, des familles originaires de Samsun et Trabzon, région de la mer Noire). Mais on observe, à l'inverse, des recompositions intra-ethniques postmigratoires ${ }^{(10)}$. Ainsi, les Kurdes, arrivés comme ouvriers avec les autres Turcs dans les années soixantedix, ont développé des stratégies identitaires quand les premiers réfugiés politiques sont arrivés, dans les années quatre-vingt/quatre-vingtdix. De même, des alévis, minorité religieuse d'obédience chiite ${ }^{(11)}$, présents dans les différentes villes, ont commencé à s'identifier clairement comme tels à Quimper, en créant une association. Globalement, d'ailleurs, le mouvement associatif turc en Bretagne est très structuré. Une fédération de l'Ouest réunit aujourd'hui toutes les associations

10)- Voir Anne Guillou et Martine Wadbled, Migrations turques en Bretagne. Rennes, Vannes, Quimper, rapport, Rennes, Addras-Fasild, juin $2004,161 \mathrm{p}$. (téléchargeable sur www.addras.com).

11)- Minoritaire en Turquie, l'alevisme, l'est aussi dans la migration. C'est l'islam sunnite qui constitue la religion très largement majoritaire. locales de travailleurs turcs.

La localisation résidentielle des immigrés turcs et de leurs descendants s'inscrit dans un processus connu d'installation des migrants. Ils ont d'abord été logés, et habitent encore pour la majorité, dans les logements sociaux de quartiers périphériques des villes : appartements d'habitats collectifs adaptés à la composition de familles comportant souvent plus de cinq enfants. Les besoins en grands logements, ainsi qu'une cer- 
12)- Contrairement aux représentations qu'en ont souvent les habitants des quartiers, les familles issues de Turquie restent très minoritaires sur le plan quantitatif. taine volonté de rapprochement résidentiel des familles (favorisant l'entraide pour la garde des enfants, les courses, par exemple) ou des amis (facilité de visites pour les personnes âgées ou sans permis de conduire) ont entraîné une relative densité de ces populations sur un même territoire résidentiel et avec elle, une plus grande visibilité(12). Le port de foulards et de grands manteaux chez certaines femmes est souvent perçu de façon négative par les autres habitants du quartier et, d'une façon générale, les personnes turques interviewées se sentent victimes de discrimination et de représentations négatives, voire hostiles, surtout les femmes. Mais, avec la durée du séjour, le rapport à l'habitat et au voisinage évolue et, depuis la fin des années quatre-vingt-dix, des familles en ascension sociale ainsi qu'un nombre croissant de jeunes couples pour lesquels l'établissement en France est définitif quitte ces quartiers et le cocon familial pour accéder à la propriété d'une maison individuelle avec jardin dans un lotissement. Cette tendance est surtout marquée à Quimper où les prix du terrain sont moins prohibitifs (une moyenne de quinze demandes de permis de construire "turcs" par an de 2001 à 2003 pour une moyenne annuelle totale de quatre cent vingt environ).

\section{Des parcours chaotiques soumis aux crises du bâtiment}

Les premiers migrants turcs sont venus en Bretagne à l'invitation d'entreprises françaises du bâtiment qui, dans les années soixante-dix, étaient demandeuses de main-d'œuvre et recrutaient dans le cadre d'accords économiques bilatéraux entre la France et la Turquie - ce type d'entente existait d'ailleurs aussi entre plusieurs autres pays du Nord et du Sud. Au cours de ce recrutement, dont les premières phases se déroulaient en Turquie, certains travailleurs avaient demandé à partir en Allemagne, réputée offrir un meilleur niveau de vie, mais l'Allemagne avait alors déjà ralenti sa demande de main-d'œuvre et la France prenait le relais, via ses entreprises, en alimentant en ouvriers étrangers, d'abord ses grands bassins industriels, puis ses régions plus rurales. Ces hommes, jeunes mais souvent déjà mariés au pays, qui sont arrivés en Bretagne ont eu des parcours tout de suite marqués par une grande précarité des contrats de travail et des conditions de vie. Débutant souvent comme jeunes ouvriers chez un patron français, ils se déplaçaient beaucoup (car la paye était meilleure) y compris à l'étranger, notamment dans les pays du Golfe. Quand les entreprises fermaient, ils retrouvaient assez facilement un emploi dans une autre société du même secteur, du moins jusque dans les années quatre-vingt. Le registre d'entrées du foyer de travailleurs Guy Houist de Rennes, qui ouvre alors ses portes, témoigne des va-et-vient incessants de ces hommes allant de chantiers en chantiers. Ainsi, entre 1975 et 1994, 37 \% des travailleurs turcs y effectuent plus d'un séjour, quittant Rennes et y revenant. 
Parmi tant d'autres, le récit biographique qui suit, celui d'un ouvrier maçon âgé de 56 ans, résume ces parcours chaotiques très soumis à l'état de santé du marché du bâtiment : "En 1974, j'ai été à Chamonix. J'avais 28 ans, j'étais déjà marié et j'avais trois enfants. J'ai passé un an à Chamonix. Après, je suis parti à Caen avec le même patron. L'entreprise payait la nourriture et le logement. Je suis resté à Caen pendant deux ans et demi. Puis à Alençon, cette fois avec une autre société, la GTB, pendant un an. Enfin, en 1979, je suis arrivé à Rennes, pour la société Catelli. J'y suis resté pendant trois ans. J'ai été tout de suite au foyer Guy Houist en arrivant. Dans l'entreprise, il y avait des travailleurs marocains, arabes, français. Je n'avais pas de contrat [c'est-à-dire qu'il avait un contrat à durée indéterminée]. Mais à la fin du chantier, c'était fini, il fallait trouver autre chose. Pour la paye, oui, ça allait. Je suis donc resté trois ans chez Catelli, puis à la Sotraco [qui n'existe plus aujourd'hui]. Le père, patron de la Sotraco est mort et le fils a repris. J'y suis resté un an, à Cherbourg."

Dans les années quatre-vingt, deux évolutions ont changé la donne, parce qu'elles ont modifié tout à la fois les conditions de vie et la perception de l'avenir migratoire. D'abord le regroupement familial qui a amorcé l'installation durable dans le pays d'accueil et a rendu obsolètes les déplacements professionnels incessants et la vie entre hommes dans les baraquements des chantiers. Mais c'est surtout la crise du bâtiment, récurrente et cyclique, occasionnant la fermeture de nombreuses petites et moyennes entreprises bretonnes dans les années quatre-vingt/quatre-vingt-dix, qui est à l'origine du mouvement qui s'est alors amorcé chez les Turcs vers la création de petites sociétés artisanales. Ce phénomène de création d'emplois indépendants, selon une enquête française récente, est perceptible chez plusieurs autres populations immigrées puisqu'aujourd'hui, en France, $15 \%$ des travailleurs indépendants sont nés à l'étranger ${ }^{(13)}$.

\section{Courageux, travailleurs, endurants}

Les anciens salariés devenus artisans demeurent sous-traitants de grandes entreprises françaises du bâtiment et trouvent facilement de l'ouvrage, toujours dans cette spécialité qu'ils ont faite leur en arrivant en Bretagne, celle de la pose de parpaings. Au fil des ans, ces ouvriers turcs se sont globalement taillé une réputation de travailleurs courageux et compétents. Ils n'hésitent d'ailleurs pas à reprendre à leur compte les ethnotypes développés à leur sujet : "fort comme un Turc" ne serait pas un vain mot, à entendre certains interviewés ! La très grande valorisation du travail, de l'endurance, de la résistance physique, du courage apparaissent dans de nombreuses interviews de Turcs, hommes ou femmes. La comparaison est souvent faite en défaveur des Français qui, eux, tiendraient moins le coup sur les chantiers
13)- Salvatore Palidda "Le développement des activités indépendantes des immigrés en Europe et en France", Revue européenne des migrations internationales, 2004, 8, 1 , p. 83-96. 
14)- Jacques Fremontier, La vie en bleu, voyage en culture ouvrière Fayard, Paris, 1980. ou dans les champs et, surtout, "travaillent en regardant leur montre" alors que leurs homologues turcs se dépenseraient sans compter. "Je me rappelle à Romorantin, nous raconte une femme de 31 ans, grandie en France, mariée et femme au foyer, qui a longtemps travaillé dans l'agriculture, on était vraiment bosseurs. On faisait vraiment le travail jusqu'au bout et on ne laissait pas tomber. Alors que les Français, ils restaient deux-trois jours et ensuite, on ne les revoyait plus. Je ne sais pas pourquoi. Nous, ce n'était pas comme ça. Peutêtre aussi parce qu'on avait l'habitude du travail dans les champs en Turquie. Le patron était content de nous." Cette grande valorisation du travail n'est pas spécifique à nos interviewés, et elle est surtout caractéristique de la culture ouvrière ${ }^{(14)}$, mais elle prend ici une coloration ethnique et joue une fonction importante dans la constitution de l'enclave ethnique, en tant que stéréotype ethnique positif.

Linscription comme artisan à la chambre de commerce procède d'une logique qu'il faut décrire en détail si l'on veut comprendre comment s'articulent marché du travail, stratégie d'insertion socio-économique d'un groupe de migrants globalement stigmatisés en France aujourd'hui - et la Bretagne, avec des nuances locales que nous ne pouvons détailler ici, n'échappe pas à ce phénomène - et ethnicisation des rapports économiques. Le phénomène est donc à double facette, celle, externe, de l'organisation interethnique de l'enclave et celle, interne, de la gestion intra-ethnique de la précarité.

\section{Artisans, le mirage de la promotion}

Devenir artisan représente - ou a représenté au début -, pour les maçons turcs interviewés comme pour beaucoup d'ouvriers quelle que soit leur origine ethnique, une promotion sociale au regard de la position de salarié. Mais, dans les faits, le statut de "patron" s'est vite avéré assez illusoire. D'abord, ces artisans turcs du bâtiment travaillent souvent seuls ou avec peu d'ouvriers salariés. Selon les chiffres de la chambre des métiers d'Ille-et-Vilaine concernant le Blosne, quartier où réside une forte proportion des Turcs de la ville, parmi les 43 entreprises artisanales turques recensées, aucune n'a plus de trois salariés et un quart n'a même aucun salarié. Le maçon turc artisan du bâtiment ressemble donc beaucoup à l'ouvrier salarié qu'il était il y a peu, d'autant qu'il travaille toujours pour les mêmes entreprises donneuses d'ordre. Comme le résume cette personne : "Je me suis installé comme artisan. D'un côté c'est mieux parce que c'est toi-même qui choisis le chantier mais d'un autre côté, tout ce qui est État, on paye les charges. Je faisais de la sous-traitance au mètre carré pour une entreprise française : coffrage et parpaings. Il y avait des délais à respecter, sinon, on était pénalisé de $5 \%$ si on dépassait le délai. Côté santé [cet homme est aujourd'hui en congé maladie de longue durée], le travail 
[salarié] pour les Français, c'est des heures fixes. À cinq heures, c'est fini. Mais quand on est artisan, c'est mieux financièrement."

La question du revenu, évoquée par ce témoin, est en réalité à double tranchant. Elle mérite qu'on s'y attarde car elle est révélatrice de la façon dont les acteurs de l'économie bretonne ethnicisent la fluctuation du marché du bâtiment. En effet, ces revenus globalement plus élevés ne peuvent en fait être obtenus, le plus souvent, qu'au prix d'un jeu complexe des artisans turcs avec la législation et l'attitude ambiguë des institutions françaises.

Remarquons d'abord que la durée de vie des entreprises turques est très faible. À Quimper par exemple, du

\begin{tabular}{|l|}
\hline $\begin{array}{l}\text { Devenir artisan représente } \\
\text { une promotion sociale au regard } \\
\text { de la position de salarié. }\end{array}$ \\
\hline $\begin{array}{l}\text { Mais, dans les faits, le statut de "patron" } \\
\text { s'est vite avéré assez illusoire. }\end{array}$
\end{tabular}
$1^{\mathrm{er}}$ janvier 2000 au 10 décembre 2001, six liquidations judiciaires ont été prononcées dans le canton 3 (quartier de Kermoysan à forte concentration "ethnique") dont cinq concernaient des entreprises turques, la plupart, quatre, en SARL. Il s'agissait d'entreprises très jeunes, dont aucune n'avait plus de six ans d'âge. À Vannes, ces entreprises sont tout aussi fragiles si l'on juge par la jeunesse de celles encore en activité en 2003 : aucune des cent soixante-six entreprises artisanales turques n'a été créée avant $1998^{(15)}$ et $70 \%$ ont deux ans ou moins. Les revenus, globalement plus élevés, des artisans par rapport aux salariés sont donc partiellement illusoires car ils ne sont pas obtenus dans la pérennité d'une activité stable mais, au contraire, dans les processus de fermeture d'entreprises ou, pire, de liquidations judiciaires.

\section{Têtes de Turcs du bâtiment}

Ces faillites, dont certaines sont réputées frauduleuses ${ }^{(16)}$, cristallisent les situations de tensions interethniques et exacerbent la stigmatisation à l'encontre des migrants turcs. Certains Français, représentants d'organismes professionnels, tiennent des propos hostiles et globalisant pour décrire ce problème bien réel. L'extrait suivant permet de comprendre comment fonctionne cette ethnicisation : "On a de gros problèmes avec cette population. Ils nous laissent des ardoises énormes. Ils créent une société ici, ils vivent en noyaux. Ils sont très organisés. Ils s'inscrivent comme artisans même quand ils n'ont pas de diplômes, après trois ans [on peut s'inscrire comme artisan sans être titulaire d'un CAP si on justifie de trois ans d'expérience professionnelle]. Ils ne payent pas la TVA, pas les charges sociales. Et puis [une fois leur entreprise fermée] ils s'inscrivent au chômage et au RMI. Ils savent mieux comment fonctionne notre système que nos petits Français artisans. Tout le monde est au courant [de ces actes]. Dans
15)- D'après des informations recueillies à la chambre des métiers et de l'artisanat du Morbihan. Chiffres de 1998 au $1^{\text {er }}$ novembre 2003.

\author{
16)- La faillite frauduleuse \\ consiste à organiser \\ sa propre insolvabilité \\ pour ne pas régler \\ ses dettes, et en particulier \\ ses cotisations sociales.
}


17)- Voir Anne Guillou et Martine Wadbled, op. cit le bâtiment, il y a de moins en moins de Français. Cela dit, il y en a qui sont très bien [...] Quand ils font le stage de préparation obligatoire [pour s'inscrire comme artisan], certains viennent et ne parlent pas un mot de français. Il y a des jeunes filles de 18 ans qui viennent s'inscrire comme chefs d'entreprise. Dans la même journée, on voit le cousin, l'oncle, etc., venir s'inscrire." On observe clairement ici la transition, de l'énoncé sur le défaut de paiement des cotisations à un énoncé sur l'opacité ethnique et, finalement, le fameux "repli communautaire". Ce type de discours ethnicise une fraude et une pratique finalement assez connue dans le monde du bâtiment et, plus généralement, du travail indépendant, même s'il est possible que les artisans turcs, plus soumis à la précarité des marchés que les Français, se soustraient plus souvent que les autres aux paiements des cotisations. Quoi qu'il en soit, ce discrédit ethniste nuit à la majorité des maçons turcs comme en témoigne une comptable française dont une grande partie de la clientèle est turque : "J'en parle avec mes clients turcs. Ils ne sont pas d'accord avec ceux qui ne payent pas. Ça met la zizanie entre eux."

Or ces "têtes de Turcs" du bâtiment breton font les frais d'une situation que d'autres acteurs français, plus pragmatiques, reconnaissent comme permettant, malgré son caractère insatisfaisant pour tous les acteurs, de maintenir des prix bas à la construction, dans un secteur où la main-d'œuvre française est difficile à trouver. "Les Turcs travaillent pour des pavillonneurs en sous-traitance, à prix tirés. Beaucoup travaillent au noir. Mais ils font un boulot que les autres ne feraient pas. Ils travaillent plus que les autres. Quand ils se seront fait un peu d'argent, ils ralentiront. Ils dérangent les artisans français parce qu'ils sont payés moins cher. Mais il n'y a pas de guerre de tranchée", affirme un représentant d'une institution française en lien avec le bâtiment.

Entre stigmatisation et pragmatisme, les avis français divergent donc sur les raisons de la vulnérabilité des entreprises artisanales turques, selon la couleur politique des protagonistes mais aussi l'état du marché local, les politiques locales et les représentations plus ou moins positives des Turcs dans la population - et les trois villes divergent de ce point de vue car les Turcs jouissent d'une bien meilleure image à Quimper, pour des raisons que nous ne pouvons développer ici(17).

\section{La fragilité des entreprises}

Les maçons turcs interrogés partagent souvent, bien sûr, avec l'ensemble du milieu artisan, l'hostilité à l'égard des "charges sociales trop lourdes". Mais ils attribuent aussi la fragilité de leurs entreprises à leur méconnaissance des lois, la faiblesse de l'organisation de leur comptabilité et, en particulier, leur difficulté à gérer la transition que représente la période où s'arrêtent les aides à la création d'entreprise. Ils reconnaissent aussi leur mauvaise organisation et soupirent devant le 
nombre de papiers à rassembler pour les fournir à leurs comptables, ces derniers ne jouant pas toujours auprès d'eux le rôle pédagogique qu'ils devraient ou pourraient jouer. De ce point de vue, l'insuffisance de la formation reçue à la chambre des métiers - formation obligatoire et payante - est patente pour beaucoup d'acteurs, français comme turcs.

La seconde raison majeure de la fragilité de leurs entreprises tient aux prix tirés à la baisse par la concurrence. Celle-ci est forte, surtout dans les moments de compression du marché, où les maçons turcs, surtout les plus précaires et les derniers arrivés, sont plus exposés que leurs collègues français, beaucoup mieux payés. Cette concurrence est activée par les donneurs d'ordre et peut mener à des prix (payés au mètre linéaire de parpaings posés) qui, objectivement, ne permettent pas de gérer sainement une société artisanale : "On ne s'en sort pas, tous les mois il y en a un quifait faillite. J'ai déposé le bilan deux fois. Les impôts augmentent et les prix de la main-d'œuvre baissent. On ne dure pas longtemps", explique un maçon de 35 ans à Vannes. Dans ce cas, les micro-entreprises les plus faibles mettent la clé sous la porte et patrons et salariés s'inscrivent au RMI ou au chômage. Les prestations chômage servent finalement à assurer la césure dans les périodes difficiles et donc, en quelque sorte, à équilibrer le marché de la construction!... Les plus pragmatiques des interlocuteurs français s'en rendent bien compte et considèrent cela comme un mal nécessaire : "Si on s'inscrit trop dans un cadre légal, dit l'un d'eux, on fait exploser le système", et finalement "Ça arrange tout le monde !", affirme le représentant d'une institution française en lien avec le travail social.

La situation s'est manifestement dégradée ces dernières années à cause de la multiplication des travailleurs dans l'enclave, comme le résume, parmi beaucoup d'autres, cet artisan de 38 ans : "Le marché $d u$ bâtiment est plus dur qu'à mon arrivée [en 1990]. En 90, il y a eu une crise du bâtiment. En 91-93, ça allait puis il y a eu encore une crise. Maintenant, il y a du travail mais on travaille gratuitement. Par exemple, pour une maison moyenne, on faisait le gros œuvre à 10000 francs [1 524 euros]. Maintenant, on le fait à 8000 francs [1 219 euros]. Le prix du parpaing est passé de 6 francs [0,91 euro] à 5,50 francs [0,84 euro]. À la question "Pourquoi les prix ont-ils baissé ?" la réponse est la suivante : "Les Français sont malins et les Turcs sont jaloux." M. X. était le premier artisan turc à Quimper. Puis les autres Turcs ont voulu se mettre à leur compte. Les Français ont alors fait jouer la concurrence en lui disant: " $S$. [nom d'un artisan turc] travaille à 50 francs [7,62 euros], pourquoi tu travailles à 60 francs [9,14 euros] ?" Finalement, beaucoup d'acteurs, turcs comme français, suggèrent que les prix pratiqués encouragent la malhonnêteté, car ceux qui cassent les prix pour prendre des marchés ne sont pas capables ensuite de maintenir leur entreprise à flot dans des conditions légales et pérennes. Le travail non déclaré, notamment, pose, outre la question 
des droits sociaux des travailleurs (chômage, retraite), celle de la sécurité sur les chantiers, gravement négligée comme en attestent des accidents du travail qui semblent assez fréquents.

Les associations de travailleurs turcs ont bien essayé de prendre les choses en main, tant à Rennes qu'à Vannes et Quimper, pour discuter ouvertement de la question des prix et créer un accord entre co-ethniques sur les prix plancher. Mais ce type d'entente est illégal sur un marché libre (et les tarifs de la chambre des métiers n'ont qu'une valeur indicative et se trouvent, de fait, supérieurs aux tarifs pratiqués réellement). Aucun accord n'a pu être établi et la concurrence reste rude entre artisans turcs, d'autant que la syndicalisation des Turcs dans les organismes professionnels ne peut constituer un garde-fou car elle est faible - contrairement à d'autres régions. En revanche, plusieurs actions, destinées à améliorer la situation et le dialogue entre partenaires économiques et sociaux turcs et français ont été tentées ou sont en cours à Vannes et Rennes. (Voir article de Patrice Le Ber, page 96).

\section{Concurrence et solidarité ethnique}

Grâce à un jeu complexe de forces où interviennent les stéréotypes positifs et négatifs sur les Turcs, les tensions périodiques sur le marché, la législation sur les entreprises, les aspirations professionnelles des maçons turcs, les entreprises artisanales turques se retrouvent à jouer un rôle de tampons entre les grands constructeurs bretons sous-traitants et les fluctuations du marché. La sous-traitance permet à ces 
grandes sociétés de garder le même niveau d'embauche et d'activité tout au long de l'année, en externalisant en quelque sorte les risques liés à l'embauche. Or, seule l'organisation ethnique forte de l'activité professionnelle turque peut permettre de supporter cette précarité. En effet, ce sont les artisans eux-mêmes qui doivent, en fonction d'une demande qui peut évoluer du jour au lendemain, trouver rapidement de la main-d'œuvre ou, au contraire, débaucher. L'embauche se fait donc parfois à la journée, en cas de "coup de bourre", grâce à une "bourse au travail" informelle qui se constitue par exemple chaque matin dans une rue du quartier du Blosne à Rennes, où les artisans sont assurés de trouver des journaliers quand ils n'ont pas pu puiser dans leur propre réservoir familial et amical. Cela se fait au détriment de la déclaration du travail, car on conçoit aisément que les contrats de travail ne soient pas systématiquement à jour dans ces conditions. Une affaire a éclaté en Bretagne en 2003, où le donneur d'ordre français mis en examen a ainsi rejeté la faute sur son sous-traitant turc en faisant valoir le fait que son partenaire était responsable de la main-d'œuvre - les inspecteurs du travail ayant trouvé, sur les chantiers contrôlés, des ouvriers journaliers sans papiers. On soulignera donc l'une des caractéristiques de ce type d'enclave : elle ne peut fonctionner que grâce à une forte organisation intra-ethnique et, comme telle, elle est implicitement encouragée - ou en tout cas rendue possible par les acteurs économiques français -, mais elle n'en est pas moins, selon les circonstances et les individus concernés, stigmatisée comme un "repli communautaire".

Au-delà même de cette capacité à répondre rapidement à la demande en puisant dans un réservoir ethnique de main-d'œuvre ; audelà, également, de la concurrence rude à laquelle se livrent les maçons turcs, la solidarité intra-ethnique joue à plein. Quand une entreprise fait faillite, l'artisan sera embauché en tant que salarié par un parent (car les relations professionnelles sont souvent d'abord familiales) ou un ancien employé devenu "patron" à son tour. De plus, la constitution de l'enclave s'appuie sur - et maintient - une très forte sociabilité masculine intra-ethnique, d'ailleurs mal vue de la société bretonne globale. Car cette riche vie sociale est basée sur une certaine ségrégation sexuelle, les hommes fréquentant des endroits à eux, cafés "turcs", locaux des associations, matches de football, autant de lieux où se renforcent les amitiés, où s'affirme l'identité turque masculine et circulent les informations sur les chantiers.

Enfin, ce type d'organisation professionnelle a l'avantage très précieux, du point de vue des Turcs, de permettre l'accueil, dans les meilleures conditions, des nouveaux migrants venus de Turquie dans le cadre du regroupement familial. Les jeunes gens élevés en France, filles comme garçons, se marient en effet en grande majorité avec un conjoint de Turquie qui vient ensuite s'installer en France. La logique d'accueil de ces nouveaux migrants et, de ce fait, la logique d'entreprise qui en 
découle est souvent plus familiale qu'ethnique dans la mesure où les jeunes gendres et belles-filles, choisis avec l'aval express des parents, sont, dans une proportion non négligeable, des cousins germains (croisé ou parallèle, en filiation matri ou patrilinéaire) ou des membres plus éloignés de la famille. Ces alliances ont donc une triple fonction : on aide de cette façon un parent dans son projet migratoire - aujourd'hui rendu presque impossible selon d'autres modalités légales à cause de la fermeture des frontières françaises -, on ralentit l'acculturation des enfants élevés en France et on fournit éventuellement à l'entreprise familiale un gendre qui saura maintenir la solidarité professionnelle et reproduire l'enclave ethnique dans la maçonnerie bretonne.

\section{Sortir de la sous-traitance et se diversifier}

Le type de relation interethnique, socio-économique, qui s'est créé en Bretagne dans les années quatre-vingt/quatre-vingt-dix, entre maçons turcs et entreprises du bâtiment tend à se perpétuer, grâce aux jeunes gendres venus de Turquie, mais aussi et surtout, grâce à la très forte reproduction sociale dans les milieux ouvriers turcs bretons. Les jeunes garçons abandonnent massivement leurs études sitôt atteint l'âge légal de sortie du système scolaire et embauchent encore majoritairement dans les entreprises co-ethniques. Les ressorts de cette reproduction sont connus : modèle parental, difficulté des parents à suivre la scolarité de leurs enfants et sentiment d'illégitimité face à l'école, enseignants dirigeant prioritairement les élèves turcs vers les filières professionnelles, etc. Toutefois, deux évolutions se font jour.

La première est l'effort, réalisé par quelques enfants d'immigrés, aujourd'hui adultes, pour progresser d'un échelon au sein du secteur du bâtiment en devenant entrepreneur eux-mêmes. Jouissant d'une meilleure connaissance que leurs parents de la société française où ils ont grandi, parlant parfaitement le français - dont certains avec un accent breton -, leur but est de contourner les donneurs d'ordre français pour établir un contact direct avec le client et sortir de la soustraitance. Cela demande un important travail de recherche de clientèle qu'un petit nombre a déjà entrepris. Par ailleurs, les activités exercées au sein du bâtiment tendent à se diversifier un peu - même si les jeunes Turcs passent peu par les centres d'apprentissages et continuent à être formés sur le tas (dix élèves seulement en 2003-2004 au centre de formation des apprentis à Quimper, par exemple). Mais ceux qui suivent la formation des métiers du bâtiment se tournent, pour poursuivre avec le cas de Quimper, pour moitié d'entre eux vers la plomberie, la plâtrerie (CAP) et l'électrotechnique (Bac Pro). Carreleurs, charpentiers, électriciens, plâtriers sont des métiers qui commencent à recruter des Turcs. D'une première enclave ethnique, la tendance va donc vers les métiers de second œuvre dans le bâtiment, 
pour le moment tenus par les "Français" ou les descendants de migrants plus anciens (portugais, espagnols). Enfin, la promotion sociale est aussi envisagée comme passant par le commerce, activité qui permet également une organisation familiale et ethnique du travail mais qui est moins pénible physiquement que le bâtiment. L'ouverture d'un restaurant est le vœu formulé par plusieurs familles, même s'il s'agit plus souvent là d'un rêve exprimé que d'une réalité.

La situation particulière des maçons turcs en Bretagne met en lumière différentes facettes d'un mécanisme plus général, celui de l'ethnostratification du travail - processus par lequel les positions des acteurs économiques sur le marché sont hiérarchisées et modelées entièrement ou partiellement - par leur appartenance ethnique ${ }^{(18)}$. Comme on l'a montré ici, l'examen de ce type de hiérarchisation suppose d'étudier à la fois les caractéristiques du domaine économique local et la façon dont il intègre de nouveaux acteurs, mais également, la manière dont ces derniers s'emploient à y trouver une place. La prise en compte simultanée des versants inter et intra-ethnique permet, notamment, de comprendre l'importance des stéréotypes ethniques dans la mise en œuvre de cette stratification. Ces ethnotypes tantôt positifs ("ce sont de bons travailleurs"), tantôt négatifs ("ils vivent entre eux", "ils ne paient pas leurs cotisations") sont en effet mobilisés à des moments différents, à des fins différentes. On conçoit bien, dans ces conditions, que la fameuse question de "l'intégration" se pose de façon infiniment plus complexe qu'il y paraît car, dans le cas breton, l'intégration socio-économique finalement réussie des maçons turcs si l'on choisit comme critère la mise au travail rapide des nouveaux venus, un niveau de revenu satisfaisant, et l'accession à la propriété, au moins à Quimper et à Vannes - n'en est pas moins problématique au regard d'autres critères comme le maintien d'une grande précarité de l'emploi artisanal ou salarié et la difficulté à sortir de l'enclave ethnique, tout à la fois espace de liberté et d'enfermement.

Anne Guillou, "Postures et apparence physique khmères, du Cambodge à la France"

- Dossier France, terre d'Asie, $n^{\circ}$ 1234, novembre-décembre 2001

Martine Wadbled, "La transmission culturelle active dans les familles viêtnamiennes"

- Dossier France, terre d'Asie, n 1234, novembre-décembre 2001

Dossier Pays-de-la-Loire, divers et ouverts, n 1222, novembre-décembre 1999

18)- Les sociologues préfèrent parler d'ethnicité pour désigner cette réalité L'ethnicité désigne à la fois : 1- La dimension identitaire des individus (la façon dont eux-mêmes se perçoivent et sont perçus par les autres ; les groupes d'appartenance dont ils se réclament, auxquels ils s'identifient ; les groupes dont ils se démarquent) 2- La dimension culturelle et religieuse de ces pratiques (la façon dont ils vivent, se comportent et pensent). L'ethnicité est fortement influencée par les situations sociales et, en particulier, par les relations interethniques elles-mêmes. 